\title{
Analytical Modeling and Computer Simulation of the Transformation of Ellipsoids Nucleated on Random Parallel Planes
}

\author{
Gabriella Maria Silveira de Sáa* (D), Harison da Silva Ventura ${ }^{a}$, Weslley Luiz da Silva Assis ${ }^{a}$, \\ Elena Villa ${ }^{b}$ Paulo Rangel Rios ${ }^{a}$ (D)

\begin{abstract}
${ }^{a}$ Universidade Federal Fluminense, Escola de Engenharia Industrial Metalúrgica de Volta Redonda, Av. dos Trabalhadores, 420, 27255-125, Volta Redonda, RJ, Brasil

${ }^{b}$ University of Milan, Department of Mathematics, via Saldini, 50, 20133, Milano, Italy
\end{abstract}

Received: April 22, 2020; Revised: June 22, 2020; Accepted: Julho 26, 2020

\begin{abstract}
A considerable number of engineering materials is polycrystalline. Cahn proposed analytical expressions for transformations nucleated at the grain faces, triple junctions, and quadruple junctions. Those places are usually the places in which a new phase takes place. Cahn assumed that the new grain boundary nucleated phase grew as spheres. Nonetheless, the austenite grain boundary nucleated ferrite does not always grow as spheres. Bradley et al. demonstrated in a series of papers that a grain boundary nucleated ferrite allotriomorph is best described by an oblate ellipsoid. The reason for ellipsoidal growth is that the growth along the grain boundary plane is faster than the thickening into the austenite. Because of this, it is of interest to have analytical solutions and computer simulations of grain boundary nucleated transformations not only for spherical growth but also for ellipsoidal growth. In this work, we present a computer simulation of grain boundary nucleated transformations that grow as ellipsoids. The computer simulation results were compared with a new exact analytical expression obtained by Villa and Rios.
\end{abstract}

Keywords: microstructure, grain boundaries, analytical methods, phase transformations, ellipsoidal growth.

\section{Introduction}

The initial work of Kolmogorov ${ }^{1}$, Johnson-Mehl' ${ }^{2}$, and Avrami $^{3}$, known as KJMA theory, considered that the nucleation sites were uniform randomly distributed in space. In KJMA work, the spatial distribution of the nuclei was the same for the two different nucleation rates they used. In the first, the initial nucleation rate was extremely high at the beginning of the transformation, with no nucleation taking place afterward. This is the so-called site-saturation, and it will be employed in the present paper. The well-known expression for the site-saturated case is given here for convenience, as this work will focus on site-saturated nucleation

$$
V_{V}(t)=1-\exp \left(\frac{-4 \pi}{3} \lambda G^{3} t^{3}\right)
$$

In Equation $1 V_{V}(t)$ is the volume fraction transformed as a function of time, $t$. The growth velocity is a constant, $G$. The number of nuclei per unit of volume is $\lambda$. It is worthy of note that nuclei were uniform randomly distributed in space in both cases. Equation 1 applies to the description of a transformation within a homogeneous matrix.

In the second case, the nucleation rate was supposed to be constant. This is the so-called constant nucleation rate transformation.

*e-mail: gabriellasilveira@id.uff.br
In a classical paper, Vandermeer and Rath ${ }^{4}$ demonstrated that the recrystallization kinetics of an iron single crystal could be described by the site-saturation transformation in agreement with KJMA.

In practice, many situations arise that differ from JMAK's original assumptions. Perhaps the easiest deviation is that the velocity might not be constant during transformation. In this particular case, JMAK findings can be adapted by introducing, for example, a time-dependent velocity. JMAK methodology may not be so straightforward to modify in other cases. There might be situations in which the nucleation rate and growth rate may not be easy to determine. Avrami herself recognized that deviations from their assumptions could take place and proposed a generalized form of her equation that is known to this day as Avrami's equation ${ }^{11}$

$$
V_{V}(t)=1-\exp \left(-k t^{n}\right)
$$

where $k$ and $n$ are adjustable parameters.

More recently, Rios and Villa generalized KJMA's theory using Stochastic Geometry ${ }^{5,6}$. The concept of homogeneous Poisson point process replaced the idea of uniform randomly. Rios and Villa showed how to extend KJMA's model to the situation in which nuclei were located in space according to an inhomogeneous Poisson point process. More details can be found in ref. 5 . However, the nucleation sites can be located 
in space in many different ways. Rios and Villa derived exact expressions for some of those cases, e. g. refs. ${ }^{5,7,8}$.

Polycrystals are essential engineering materials. In polycrystals, the nucleation usually takes place on the grain boundaries or faces, on the triple junctions or grain edges, and on the quadruple junctions or the grains vertices. $\mathrm{Cahn}^{9}$ treated the grain faces as planes randomly located in space, grain edges as lines also randomly located in space, and grain vertices as points uniform randomly located in space. Therefore, Cahn's expression for nucleation on the grain vertices coincided with those provided by the KJMA theory, Equation 1.

The situation that this paper examines more closely is sitesaturation nucleation on grain faces. Specifically, Cahn derived an expression for the kinetics of transformations nucleated on grain boundaries. As said above, Cahn supposed that the grain boundaries were random planes. Cahn's expression has been evaluated against computer simulations. The results show that the expression is not entirely accurate, depending on the underlying network representing the polycrystal ${ }^{10,11}$. Still, Cahn's equation is the only analytical expression available. Jägle et al. ${ }^{12}$ suggested that Cahn's equation may be useful to describe a wide range of situations if one can treat one of its parameters as an adjustable parameter.

Cahn supposed that the nuclei grew with a spherical shape. For grains growing with a spherical shape, Rios and Villa $^{13,14}$ demonstrated that Cahn's expression for random planes was identical to the expression derived for parallel planes. The point is that Cahn's assumption of random planes in space is not necessary. The assumption that the number of random planes, $N$, in a random volume must be a Poisson random variable is enough to yield Cahn's expression ${ }^{13,14}$. For instance, for random parallel planes, the only requirement is that the number of parallel planes in a random volume must be a Poisson random variable. A detailed comparison between Cahn's methodology and the rigorous mathematical treatment of Rios and Villa can be found in ${ }^{13,14}$.

The assumption of spherical growth is standard. Nonetheless, new regions do not always grow as spheres ${ }^{4,15-17}$. Bradley et al. demonstrated in a series of papers ${ }^{18-20}$ that a grain boundary nucleated ferrite allotriomorph is best described by an oblate ellipsoid. The reason why these regions grow with ellipsoidal shape is that the growth on the grain boundary plane is faster than the thickening into the austenite ${ }^{18-20}$.

Moreover, Enomoto and Aaronson ${ }^{22}$ carried out a detailed study of ferrite nucleation kinetics on the grain boundaries. They found out that ferrite nuclei density at the austenite grain boundaries decreased as the nucleation temperature increases. In this work, we use the assumption of sitesaturation. Site-saturation means that the nucleation rate is so fast at the beginning of the transformation that the available nucleation sites at the grain boundary are saturated early in the transformation. Therefore, site-saturation essentially signifies that all nuclei are already present at the time origin and no nuclei form later in the transformation. Because of these classical works by Aaronson's group, it is of interest to have analytical solutions for grain boundary nucleated transformations not only for spherical growth but also for ellipsoidal growth. For instance, Tanaka et al. ${ }^{23}$ used an expression derived by Obara et al. ${ }^{24}$ based on Cahn, for the growth of oblate ellipsoids of aspect ratio 1/3.

Moreover, we can, therefore, assume that the nuclei density on the parallel planes has different values that would correspond to different nucleation temperatures. ${ }^{22}$

Despite Aaronson's group in-depth studies of the growth of oblate ferrite regions from austenite grain boundaries, there has been no theoretical follow up to their work.

In this work, we approach the issue of generalizing ellipsoidal nucleation on grain boundaries addressing three key aspects.

The first aspect is a generalization of the nucleation and growth of ellipsoids on grain boundaries. We present here in the Mathematical Background section, the results of recent work by two of the co-authors of this paper ${ }^{21}$. In their paper, Villa and Rios present a general solution for nucleation and growth of ellipsoids on random parallel planes. Villa and Rios's paper ${ }^{21}$ is mainly written for a mathematical audience and includes rigorous proofs based on Stochastic Geometry. Therefore, to present a summary of ref. ${ }^{21}$ is convenient for the Materials Scientist.

The second aspect is a computer simulation of the nucleation and growth of oblate and prolate ellipsoids on random parallel planes and the comparison of computer simulation with the exact mathematical solution of Villa and Rios ${ }^{21}$. It is, of course, helpful to have an analytical solution. Nonetheless, analytical solutions deal with the mean value of the properties. The computer simulation allows the generation of the microstructures that are extremely useful for Materials Scientists. One can also quantify the microstructural evolution both with quantities given by the exact solution and also with parameters, like the contiguity, for which one has no available analytical solution.

The third aspect is to use the result that the solution for random parallel planes is equal to the solution for random planes, as described above. This result is valuable as it allows us to discuss the behavior of the nucleation and growth of ellipsoids on random planes, that is, grain boundaries based on the analytical solution and computer simulation for random parallel planes.

Summarizing, in this work, we simulate grain boundary nucleated transformations that grow as ellipsoids. We compare our results with a recently obtained ${ }^{21}$ general mathematical expression for the nucleation and growth of ellipsoids on parallel planes.

\section{Mathematical Background}

In what follows, we give a summary of the recently published paper by Villa and $\operatorname{Rios}^{21}$ on the problem of nucleation and growth of ellipsoids on random parallel planes. Villa and Rios is a rigorous mathematical paper with a mathematical notation that is unusual for the Materials scientist. In this summary, we only give the main results and walk the reader through mathematics. Full mathematical proofs can be found in Villa and Rios ${ }^{21}$. In the Discussion section, we talk about the applicability of this solution to random planes.

Rios and Villa have rigorously obtained explicit mathematical expressions for mean volume and surface densities of dynamical germ-grain models with spherical and ellipsoidal growth whose associated nucleation process takes place on 
random parallel planes in refs. ${ }^{13,21}$ respectively. Such papers contain more general results, which go beyond the aim of the present paper. Therefore, we recall here essential notion and results we are going to apply in the sequel, referring to the above mentioned papers for the proofs and further details.

The transformation under consideration proceeds in $\mathbb{R}^{3}$ over time in two distinct steps: in the first one, the new region appears, and in the second one, this new region increases in size. These steps are mathematically described employing the so-called birth-and-growth (stochastic) process, or, equivalently, nucleation-and-growth (stochastic) process. Namely, a birth-and-growth process is a dynamic germ-grain model used to model situations in which nuclei (germs) are born in time and are located in space randomly. Each nucleus generates a grain evolving in time according to a given growth law. Since, in general, the nucleation is random in time and space, then the transformed region at any time $t>0$ will be a random set in $\mathbb{R}^{3}\left(\right.$ e.g., see ${ }^{6}$. Denote by $T_{j}$ the $\mathbb{R}_{+}$-valued random variable representing the time of birth of the $j$-th nucleus, and by $X_{j}$ the $\mathbb{R}^{d}$-valued random variable representing the spatial location of the nucleus born at time $T_{j}$. Let $\Theta_{T_{j}}^{t}\left(X_{j}\right)$ be the grain obtained as the evolution up to time $t \geq T_{j}$ of the nucleus born at time $T_{j}$ in $X_{j}$; then the microstructure of the sample $\Theta^{t}$ at time $t$ is of the type

$$
\Theta^{t}=\bigcup_{T_{j} \leq t} \Theta_{T_{j}}^{t}\left(X_{j}\right), \quad t \in \mathbb{R}_{+}
$$

In the following we shall consider the simpler case of site-saturated nucleation; this occurs when all nuclei appear at the start of the transformation, $t=0$, but no nuclei form after that (i.e., $T_{j}=0$ for any $j$ ). Otherwise, the nucleation is referred to as time-dependent nucleation. A site-saturated nucleation process may be modeled utilizing a point process $N$ in $\mathbb{R}^{3}$, that is a locally finite sequence of points $\left\{X_{j}\right\}_{j}$ randomly located in space, according to a given probability law. An important measure associated with any point process $N$ is the so-called intensity measure $A$ of $N$ , that is a measure on $\mathbb{R}^{3}$ defined as $\Lambda(A):=\mathbb{E}[N(A)]$ for all $A \in \mathcal{B}_{\mathbb{R}^{3}}$ representing the mean number of nuclei in $A . \mathcal{B}_{\mathbb{R}^{3}}$ the Borel $\sigma$-algebra of $\mathbb{R}^{3}$.

Moreover, we shall assume that all the grains growth with ellipsoidal shape, with a fixed orientation, and that the nucleation process takes place only on parallel planes, with an orthogonal direction parallel to one of the three semi-axis of the grains. Let us denote

$$
E_{0}:=\left\{\left(x_{1}, x_{2}, x_{3}\right) \in \mathbb{R}^{3}: \frac{x_{1}^{2}}{a_{1}^{2}}+\frac{x_{2}^{2}}{a_{2}^{2}}+\frac{x_{3}^{2}}{a_{3}^{2}} \leq 1\right\}
$$

then for any $t>0$

$$
\Theta^{t}=\bigcup_{X_{j} \in N}\left(X_{j}+G t E_{0}\right)
$$

that is a random union of ellipsoids centered in the random point $X_{j}$ of the nucleation process $N$, and with semi-axes lengths given by $G t a_{1}, G t a_{2}$ and $G t a_{3}$. Notice that the spherical case follows a particular case by taking $a_{1}=a_{2}=a_{3}=1$.

To model nucleation on random parallel planes in $\mathbb{R}^{2} \times[0, K]$, we need to introduce: the nucleation process $N_{u}$ on a fixed plane $B(u):=\left\{x \in \mathbb{R}^{3}: x_{3}=u\right\}$ for any value $u \geq 0$, and the point process $\Xi=\left\{D_{i}\right\}_{i}$ on the positive $x_{3}$-semi-axis, representing the random distances from the origin of the planes $B_{1}=B\left(D_{1}\right), B_{2}=B\left(D_{2}\right), \ldots$, respectively. Then, the transformed region under consideration at time $t$, say $\Theta_{K}^{t}$, will be given by the union of the transformed regions $\Theta^{t, D_{i}}$ at time $t$ due to the nucleation on the planes $B\left(D_{i}\right)$ with random distances $D_{i} \in[0, K]$ from the origin, where for any realization $D_{i}=u_{i}$ the corresponding transformed region $\Theta^{t, u_{i}}$ is defined as in (3) by replacing $N$ with $N_{u_{i}}$.

For any fixed $u>0$ we assume that $N_{u}$ is a Poisson point process on $B(u)$ with intensity measure

$$
\Lambda_{u}(d x)=f(x) \delta_{B(u)}(x) d x
$$

where $\delta_{B(u)}(x)$ is the usual Dirac-delta function on $B(u)$, which forces the nucleation on the plane $B(u)$. Besides, we assume that even $\Xi=\left\{D_{i}\right\}_{i}$ is a Poisson process in $\mathbb{R}_{+}$, with intensity measure $\Lambda_{\Xi}(d u)=h(u)_{(0, \infty)}(u) d u$.

We also recall that the mean volume density $V_{V}(t, x)$ at point $x \in \mathbb{R}^{3}$ of $\Theta_{K}^{t}$ at time $t$ is defined to be the quantity such that

$$
\int_{A} V_{V}(t, x) d x=\mathbb{E}\left[v^{3}\left(\Theta^{t} \cap A\right)\right] \quad \forall A \in \mathcal{B}_{\mathbb{R}^{3}}
$$

where $\mathbb{E}$ denotes the expected value, $v^{3}$ the volume measure, and $\mathcal{B}_{\mathbb{R}^{3}}$ the Borel $\sigma$-algebra of $\mathbb{R}^{3}$.

It is proved in ref. ${ }^{22}$ that if the function $f$ is varying along a preferential direction, that is it of the type

$$
f(x)=\sum_{i=1}^{3} p_{i} x_{i}+q
$$

with $p \in \mathbb{R}^{3}$, and $q \in \mathbb{R}$ such that $f(x) \geq 0$ for any $x$ in the considered observation window, then

$$
V_{V}(t, x)=1-\exp \left\{-\int_{0}^{K} V_{V}^{u}(t, x) h(u) d u\right\}
$$

with

$$
V_{V}^{u}(t, x)=\left(1-\exp \left\{-f\left(\left(x_{1}, x_{2}, u\right)\right) \pi a_{1} a_{2}\left(G^{2} t^{2}-\frac{\left(u-x_{3}\right)^{2}}{a_{3}^{2}}\right)\right\}\right\}_{\left[x_{3}-G t t_{3}, x_{3}+G t a_{3}\right]}(u)
$$

Equations 9 and 10 are proved in ref. ${ }^{21}$. It is the first time an analytical model yielding the mean volume density, $V_{V}(t, x)$, rather than the volume fraction is derived. However, we will not explore this result fully in here. Only particular cases in which one has the volume fraction transformed are going to be simulated here.

Let $K=1$, that is our specimen is in $\mathbb{R}^{2} \times[0,1]$. We distinguish the following site-saturated cases: (We refer to ref. ${ }^{21}$ for a rigorous mathematical derivation.)

\section{Same homogeneous nucleation on each plane; inhomogeneous distribution of the planes}

Assume that the mean number of nuclei per unit of area on each plane is $c>0$ (that is $f(x) \equiv c$ in (6) and that $h(u)=b_{1} u+b_{0}$, with $b_{0}$ and $b_{I}$ positive; then

$$
V_{V}(t, x)=1-\exp \left\{-\int_{\max \left\{0, x_{3}-G t a_{3}\right\}}^{\min \left\{1, x_{3}+G t a_{3}\right\}} V_{V}^{u}(t, x)\left(b_{1} u+b_{2}\right) d u\right\}
$$

with 


$$
V_{V}^{u}(t, x)=1-e^{-c \pi a_{1} a_{2}\left(G^{2} t^{2}-\left(u-x_{3}\right)^{2} / a_{3}^{2}\right)}
$$

\section{Different homogeneous nucleation on each plane; homogeneous distribution of the planes}

Assume that the function $f$ is varying only along the $x_{3}$-semi-axis that is

$$
f(x)=p x_{3}+q, \quad p, q>0
$$

This implies homogeneous nucleation on each plane, increasing as the distance of the plane from the origin increases.

Assume also that the Poisson process $\Xi$ has constant intensity $h(u) \equiv M$; this means that there are $M$ planes in mean in $\mathbb{R}^{2} \times[0,1]$, and that, given that the number of planes in $\mathbb{R}^{2} \times[0,1]$ is $m$. They are uniformly distributed there.

In such a case it holds:

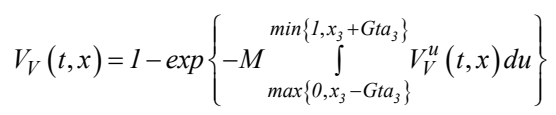

with

$$
V_{V}^{u}(t, x)=1-e^{-(p u+q) \pi a_{1} a_{2}\left(G^{2} t^{2}-\left(u-x_{3}\right)^{2} / a_{3}^{2}\right)}
$$

Same homogeneous nucleation on each plane; homogeneous distribution of the planes

By proceeding similarly as above, let $f(x) \equiv c$ and $h(u) \equiv M$; then

$$
V_{V}(t, x)=1-\exp \left\{-M \int_{\max \left\{0, x_{3}-G t a_{3}\right\}}^{\min \left\{1, x_{3}+G t a_{3}\right\}}\left(1-e^{-c \pi a_{1} a_{2}\left(G^{2} t^{2}-\left(u-x_{3}\right)^{2} / a_{3}^{2}\right)}\right) d u\right\}
$$

In the spherical case $a_{1}=a_{2}=a_{3}=1$ we reobtain as a particular case the expressions given in ref. ${ }^{4}$.

Moreover, let us observe that for any $x=\left(x_{1}, x_{2}, x_{3}\right) \in \mathbb{R}^{2} \times[0,1]$, such that $0 \leq 1-G t a_{3} \leq x_{3} \leq G t a_{3} \leq 1$, all the above integral are taken between 0 and 1 .

\section{Formulae for oblate and prolate spheroids site-saturated nucleated on random parallel planes,}

Prolate:

$$
V_{V}(t)=1-\exp \left(-2 S_{V}^{\text {planes }} a_{1} G t \int_{0}^{1}\left\{1-e^{-\pi \lambda_{s} a_{1} a_{3} G^{2} t^{2}\left[I-z^{2}\right]}\right\} d z\right)
$$

Oblate:

$$
V_{V}(t)=1-\exp \left(-2 S_{V}^{\text {planes }} a_{3} G t \int_{0}^{1}\left\{1-e^{-\pi \lambda_{s} a_{1}^{2} G^{2} t^{2}\left[1-z^{2}\right]}\right\} d z\right)
$$

Sphere:

$$
V_{V}(t)=1-\exp \left(-2 S_{V}^{\text {planes }} G t \int_{0}^{l}\left\{1-e^{-\pi \lambda_{s} G^{2} t^{2}\left[1-z^{2}\right]}\right\} d z\right)
$$

$S_{V}^{\text {planes }}$ is the mean area density per unit of volume of the planes and $\lambda_{s}$ is the number of nuclei per unit of area of a plane.

\section{Methodology}

For the nucleation and growth process, one performed a computer simulation using the Causal Cone method, ${ }^{5,25}$. The matrix comprised 500x500x500 cubic cells. Each matrix dimension was considered to have a dimensionless length equal to 1 so that the simulated domain is effectively a unit cube: $[0,1]^{3}$. Prolate ellipsoids with aspect ratios $1: 1: 8,1: 1: 4$, and $1: 1: 2$ and oblate ellipsoids with aspect ratios $8: 8: 1,4: 4: 1$ and 2:2:1 were simulated. Simulations employing spherical growth were also conducted for comparison. All simulations used site-saturated nucleation described above.

The number of nuclei and the number of planes were located within the matrix according to a homogeneous Poisson point process. There was a total of 200 nuclei within the simulation matrix for the 10 nuclei and 20 planes situation, 400 nuclei within the simulation matrix for the 20 nuclei and 20 planes situation, and 1600 nuclei within the simulation matrix for the 80 nuclei and 20 planes situation. Periodic boundary conditions were adopted. All growth velocities were kept constant throughout the transformation. Each quantity reported here is the mean value of 100 simulations. That is, we repeated each simulation 100 times. The number of repetitions has been chosen for reliable results.

\section{Results}

\subsection{Microstructural evolution}

Figure 1 shows the microstructural evolution of oblate ellipsoids on 20 random parallel planes with 20 nuclei on each plane. The ratio of the length of the longer axes to the length of the shorter axis is $4: 4: 1$. Fraction transformed to a) $V_{V}=0.1$; b) $V_{V}=0.5 ;$ c) $V_{V}=1$.

Figure 2 shows the microstructural evolution of prolate ellipsoids nucleated on 20 random parallel planes with 20 nuclei on each plane. The ratio of the length of the shorter axis to the length of longer axes of the ellipsoid is $1: 1: 4$. Fraction transformed to a) $V_{V}=0.1$; b) $V_{V}=0.5$; c) $V_{V}=1$.

Figure 3 depicts a longitudinal section of the simulation volume showing a fully transformed microstructure of oblate ellipsoids nucleated on 20 random parallel planes. The ratio of the length of the longer axes to the length of the shorter axis of the ellipsoid is $4: 4: 1$. The microstructures show the effect of the number of nuclei per plane: a) 20 nuclei per plane; b) 80 nuclei per plane.

Figure 4 depicts a longitudinal section of the simulation volume showing a fully transformed microstructure of prolate ellipsoids nucleated on 20 random parallel planes. The ratio of the length of the shorter axes to the length of the longer axis of the ellipsoid is 1:1:4. The microstructures show the effect of the number of nuclei per plane: a) 20 nuclei per plane; b) 80 nuclei per plane.

Visual inspection shows that the fully transformed microstructures generated by the oblate ellipsoids have a significant proportion of their interfacial area parallel to the plane $x_{3}=0$. Compare, for instance, with the microstructure generated by ellipsoids nucleated in space according to a homogeneous Poisson point process from our previous work ${ }^{17}$.

It is worth commenting on the microstructural feature, see arrow, in Figure $3 \mathrm{~b}$ that results from the proximity of the parallel planes. A homogeneous Poisson point process is a stochastically independent process. This means that the position of one point is independent of the other. This may result in two points or, in this case, two parallel planes being close to one another. To artificially introducing a minimum distance would violate the stochastic independence of the 


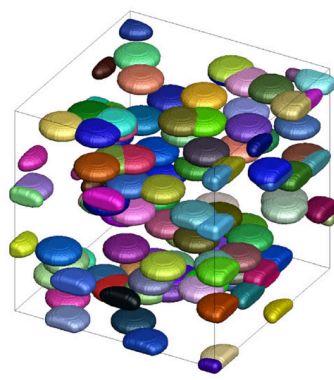

(a)

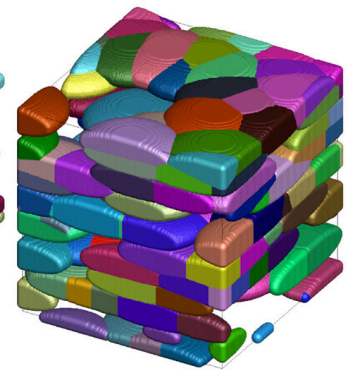

(b)

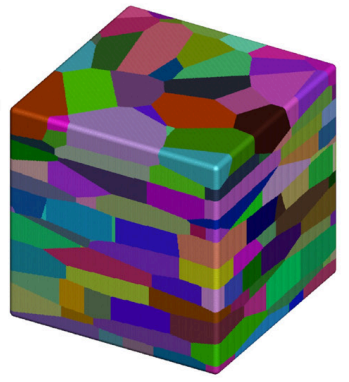

(c)

Figure 1. Microstructure of oblate ellipsoids nucleated on 20 random parallel planes with 20 random nuclei on each plane. The nucleation was site-saturated. That is, all nuclei started to grow at the time origin: $t=0$. The ratio of the length of the longer axes to the length shorter axis of the ellipsoid is 4:4:1. Each figure depicts the microstructure corresponding to a progressively higher fraction transformed, $V_{V}$ : a) $V_{V}=0.1$; b) $V_{V}=0.5$; c) $V_{V}=1$.

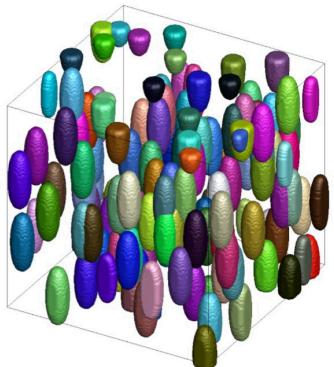

(a)

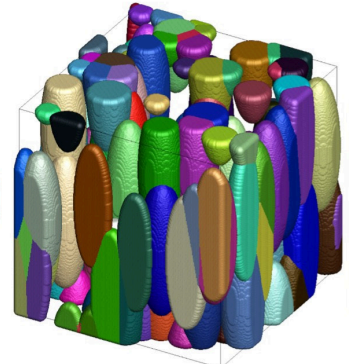

(b)



(c)

Figure 2. Microstructure of prolate ellipsoids nucleated on 20 random parallel planes with 20 random nuclei on each plane. The nucleation was site-saturated. That is, all nuclei started to grow at the time origin: $t=0$. The ratio of the length of the shorter axes of the ellipsoid the length longer axis to is 4:4:1. Each figure depicts the microstructure corresponding to a progressively higher fraction transformed, $V_{V}$ : a) $V_{V}=0.1$; b) $V_{V}=0.5$; c) $V_{V}=1$.

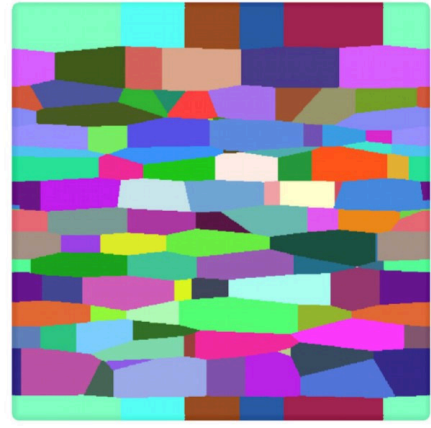

(a)

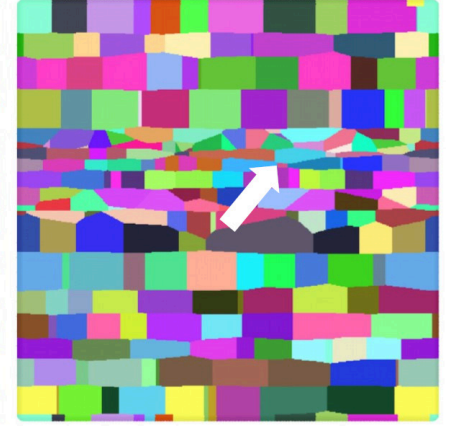

(b)

Figure 3. Longitudinal section showing the fully transformed microstructure of oblate ellipsoids nucleated on 20 random parallel planes. The ratio of the length of the longer axes to the shorter axis of the ellipsoid is $4: 4: 1$. The nucleation was site-saturated. That is, all nuclei started to grow at the time origin: $t=0$. The figures depict microstructures that show the effect for the number of nuclei per plane: a) 20 nuclei on each plane; b) 80 nuclei on each plane.

Poisson point process, and the analytical equations would not be valid anymore. Nonetheless, this is similar to the situation studied by Ventura et al. ${ }^{26,27}$ Ventura et al. studied the effect of an exclusion zone around each nuclei using convenient non-Poissonian point processes.

\subsection{Volume fraction transformed against time}

Figure 5 depicts the transformation kinetics of oblate ellipsoids nucleated on 20 random parallel planes. The ratio of the length of the longer axes to the length shorter axis of the ellipsoid is 4:4:1. The curves depicted in Figure 5 show the effect of the number of nuclei per plane on the transformation: 5a) 10 nuclei per plane; 5b) 80 nuclei per plane.

Figure 6 depicts the transformation kinetics of oblate ellipsoids nucleated on 20 random parallel planes. The curves 




(a)

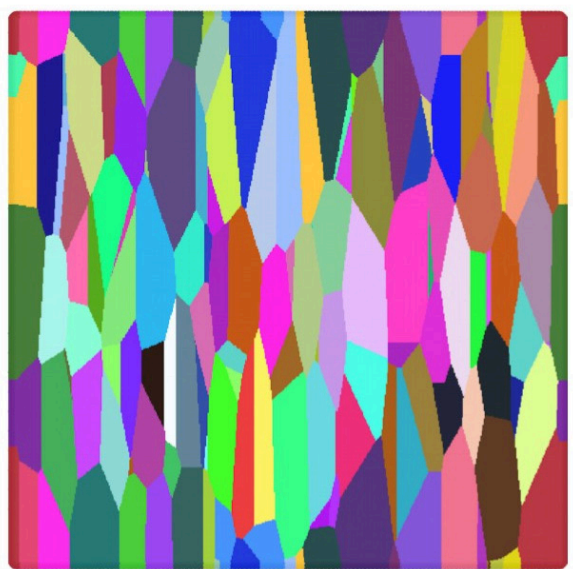

(b)

Figure 4. Longitudinal section showing the fully transformed microstructure of prolate ellipsoids nucleated on 20 random parallel planes. The ratio of the length of the shorter axes of the ellipsoid the longer axis of the ellipsoid is 1:1:4. The nucleation was site-saturated. That is, all nuclei started to grow at the time origin: $t=0$. The figures depict microstructures that show the effect of the number of nuclei per plane corresponding: a) 20 nuclei on each plane; b) 80 nuclei on each plane.



(a)

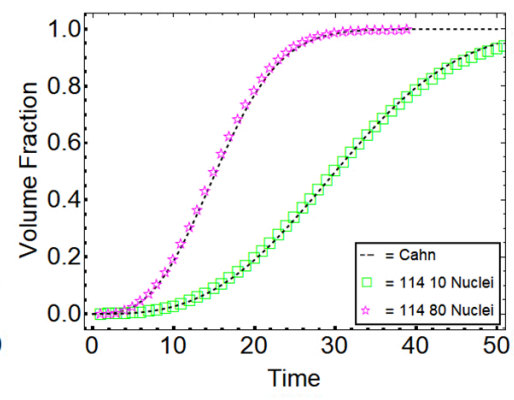

(b)

Figure 5. Transformation kinetics of oblate ellipsoids nucleated on 20 random parallel planes. The nucleation was site-saturated. That is, all nuclei started to grow at the time origin: $t=0$. The symbols correspond to the computer-simulated result, whereas the dashed line was calculated using Cahn's model modified for ellipsoids. Each figure shows the effect of the number of nuclei per plane on the transformation kinetics: 10 nuclei and 80 nuclei on each plane. Figure 5a depicts the result of the number of nuclei per plane on the transformation kinetics of oblate ellipsoids. The ratio of the length of the longer axes to the length of the shorter axis of the oblate ellipsoid is 4:4:1. Figure $5 \mathrm{~b}$ depicts the result of the number of nuclei per plane on the transformation kinetics of prolate ellipsoids. The ratio of the length of the shorter axes of the prolate ellipsoid to the longer axis is 1:1:4.

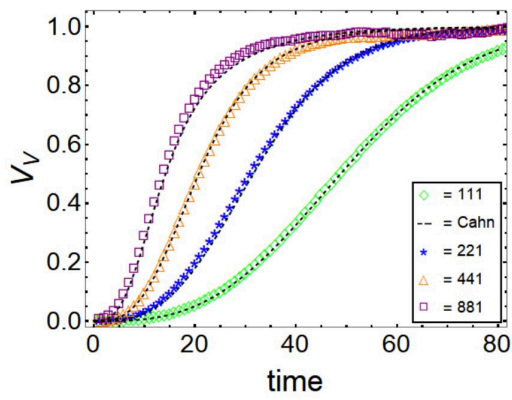

(a)

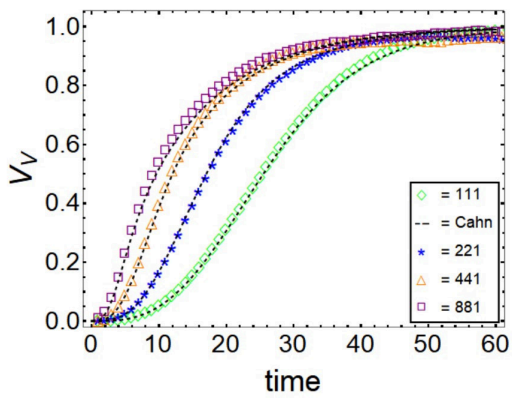

(b)

Figure 6. Effect of the aspect ratio and the number of nuclei per plane on the transformation kinetics of oblate ellipsoids. The nucleation was site-saturated. That is, all nuclei started to grow at the time origin: $t=0$. The symbols correspond to the computer-simulated result, whereas the dashed line was calculated using Cahn's model modified for ellipsoids. On each figure, the curves show the effect of the ratio of the length of the longer axes to the shorter axis of the ellipsoids. Figure 6a depicts the transformation kinetics of oblate ellipsoids nucleated on 20 random parallel planes with 10 nuclei per each plane. Figure 6a depicts the transformation kinetics of oblate ellipsoids nucleated on 20 random parallel planes with 80 number of nuclei per plane. 
show the effect of the ratio of the length of the longer axes to the length of the shorter axis the ellipsoid. The number of nuclei per plane is 6a) 10 nuclei per plane; 6b) 80 nuclei per plane.

Figure 7 depicts the transformation kinetics of prolate ellipsoids nucleated on 20 random parallel planes. The curves show the effect of the ratio of the length of the shorter axes to the longer axis the ellipsoid. The number of nuclei per plane is 7a) 10 nuclei per plane; 7b) 80 nuclei per plane.

Figures 5-7 show excellent agreement between Cahn's model modified for ellipsoids, Equation 17 and 18, with computer simulation. This agreement was expected if the simulation is correct as Equation 17 and 18 are exact. In the discussion section, we consider the factors that are essential in this kind of simulation to ensure that the simulation gives an accurate result.

As mentioned above, Figures 5-7 mainly illustrate the effect of two factors: 1 - the ellipsoid shape, oblate against prolate, Figure 5; 2 - the effect of the number of nuclei Figures 5-7. Figure 5 demonstrates that the effect of a decrease in the number of nuclei per plane from 80 to 10 nuclei per plane has a more substantial effect on the transformation kinetics than the shape of the ellipsoid, oblate, Figure 5a, or prolate, Figure 5b. Figures 6 and 7 show the effect of an increase in the number of nuclei per plane from 10, Figures $6 a$ and $7 a$, to 80 nuclei per plane, Figures $6 \mathrm{~b}$ and $7 \mathrm{~b}$. The transformation kinetics is significantly faster when one has more nuclei per plane. These effects are intuitively expected. A more interesting effect of the number of nuclei is its effect on the contiguity shown in the next section.

\subsection{Contiguity}

The contiguity is a useful metallographic parameter. Nevertheless, the contiguity is not employed very often, maybe because it requires the measurement of both the mobile (new phase - parent matrix) interfaces and immobile( new phase - new phase) interfaces. Vandermeer gives an insightful view of the contiguity backed by experimental results ${ }^{28}$. In a computer simulation, the calculation of the contiguity is straightforward. One can found that the contiguity has been used in several computer simulation papers, some of which are listed here. ${ }^{16,17,26,27,29-31}$

The contiguity, $C_{\beta \beta}$, is defined as $C_{\beta \beta}=\frac{2 S_{V}^{\beta \beta}}{S_{V}^{\alpha \beta}+2 S_{V}^{\beta \beta}}$,

where $\beta$ is a new phase, and $\alpha$ is the parent phase. $S_{V}^{\beta \beta}$ is the mean interfacial area density of the boundaries between the regions of the new phase and $S_{V}^{\alpha \beta}$ is the mean interfacial area density of the boundaries between the regions of the new

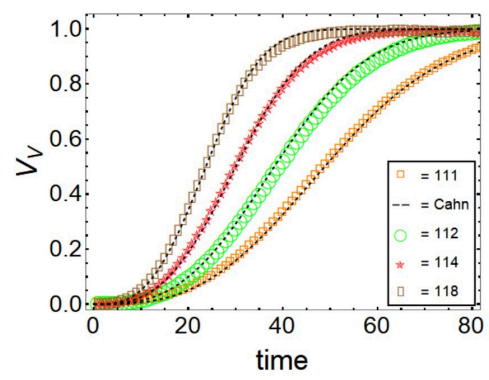

(a)

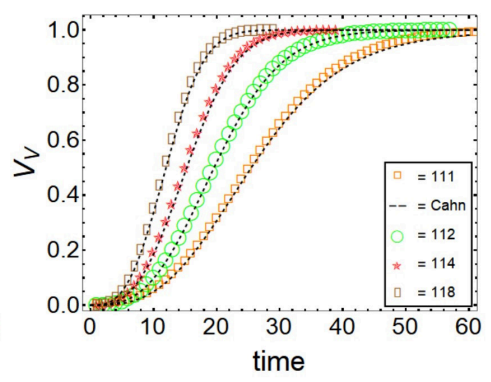

(b)

Figure 7. Effect of the aspect ratio and the number of nuclei per plane on the transformation kinetics of prolate ellipsoids. The nucleation was site-saturated. That is, all nuclei started to grow at the time origin: $t=0$. The symbols correspond to the computer-simulated result, whereas the dashed line was calculated using Cahn's model modified for ellipsoids. On each figure, the curves show the effect of the ratio of the length of the longer axes to the shorter axis of the ellipsoids. Figure 7a depicts the transformation kinetics of prolate ellipsoids nucleated on 20 random parallel planes with 10 nuclei per each plane. Figure 7a depicts the transformation kinetics of prolate ellipsoids nucleated on 20 random parallel planes with 80 number of nuclei per plane.

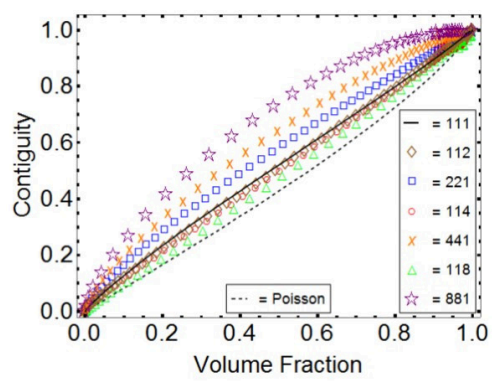

(a)

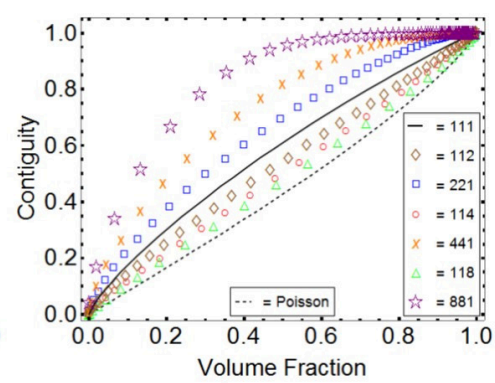

(b)

Figure 8. Contiguity of oblate and prolate ellipsoids as a function of volume fraction transformed. The curves show the effect of the ellipsoid shape and the number of nuclei per plane on the contiguity. The dashed line is the theoretical line for the contiguity of spherical regions nucleated in space according to a homogeneous Poisson point process. The solid line is the simulated contiguity of spheres nucleated on parallel planes. The symbols correspond to computer-simulated results. Figure 8a depicts the contiguity of ellipsoids nucleated on 20 random parallel planes with 10 nuclei per each plane. Figure $8 \mathrm{~b}$ depicts the contiguity of ellipsoids nucleated on 20 random parallel planes with 80 nuclei per each plane. 
phase and the parent matrix. The contiguity $C_{\beta \beta}$ is plotted as a function of volume fraction, $V_{V}(t)$.

Figure 8 depicts the contiguity of oblate and prolate ellipsoids nucleated on 20 random parallel planes. The curves show the effect of the ellipsoid shape and the number of nuclei per plane on the contiguity.

First of all, it is expedient to explain the meaning of the dashed line in more detail. The dashed line represents an exact expression for the contiguity when nucleation is site-saturated, and the nuclei are located in space according to a homogeneous Poisson point process ${ }^{29}$. Vandermeer ${ }^{28}$ and also Rios et al..$^{30}$ found out that when there is clustering, the impingement takes place earlier, and the contiguity curves are above the Poisson. Moreover, as demonstrated by Ventura et al. ${ }^{26}$, if the position of the nucleation sites are arranged periodically or approaching a periodic arrangement, the impingement takes place later, and the contiguity lies below the dashed line ${ }^{26}$. When the growing regions are ellipsoids, their contiguity still falls on the dashed line provided that their nucleation sites nuclei are located in space according to a homogeneous Poisson point process ${ }^{16,17}$.

Our results show that when the ellipsoids are nucleated on parallel planes, the contiguity in all cases lies above the dashed line. This means that nucleating on parallel planes has a clustering effect. Indeed comparing nuclei uniform randomly located in space with nuclei located on parallel planes, one sees a clustering effect, as exemplified in ${ }^{28,30}$.

Regarding both Figures $8 \mathrm{a}$ and $8 \mathrm{~b}$, one can observe two factors that influence the contiguity: the shape of the ellipsoids and the number of nuclei per plane.

Oblate and prolate ellipsoids exhibit a distinct behavior. In the oblate ellipsoids, the clustering effect is much more pronounced than in the prolate ellipsoids. The contiguity behavior, as mentioned above, is related to the impingement. Earlier impingement causes a clustering effect, and the contiguity curves lie above the theoretical dashed line curve. The oblate ellipsoids have their longer axes on the plane. Therefore the oblate ellipsoids impinge much earlier than the prolate ellipsoids that have their smaller axes on the plane. The behavior displayed both in Figures $8 \mathrm{a}$ and $8 \mathrm{~b}$ reflects this. The simulated points corresponding to the oblates, e. g. 881(stars in Figs. 8a and 8b), are well above the simulated points corresponding to the oblates, e. g. 118 (triangles in Figs. 8a and 8b). This effect is stronger as the ratio of the length of the longer axis to the length of the smaller axis increases. For example, the simulated points of the 881 ellipsoids are higher than the theoretical curve than the simulated points 221 ellipsoids. Notice that even when spheres are nucleated on the parallel planes, solid lines in Figures $8 \mathrm{a}$ and $8 \mathrm{~b}$, one still has a clustering effect.

The number of nuclei per plane is also an essential factor. Increasing the number of nuclei per plane causes an earlier impingement. Of course, for a given ellipsoidal shape, the impingement will start earlier for a larger number of nuclei per plane. The difference between Figure $8 \mathrm{a}$ and $8 \mathrm{~b}$ caused by the increased number of nuclei per plane from 10 to 80 is noticeable. The effect of the number of nuclei per plane is more significant for higher ratios of the length of longer to the length of the smaller axes. Compare, for instance, the behavior of 881 ellipsoids in Figures $8 \mathrm{a}$ and $8 \mathrm{~b}$.

\section{Discussion}

Equation 9 and 10 are very recent. Villa and Rios ${ }^{21}$ derived for the first time an analytical model yielding the mean volume density, $V_{V}(t, x)$, rather than the volume fraction. Mathematical details can be found in Villa and Rios ${ }^{21}$.

First, it is necessary to discuss technical points that are essential for a correct simulation. In the section Methodology, one described the simulation and mentioned that each curve of, for example, Figure 5a and 5b is the average result of 100 simulations. However, these curves are not only the result of 100 repetitions of 20 planes and 10 nuclei per plane. It is slightly more complicated than that, and it is described now in detail. The first step is to select 20 planes. The point is that 20 is the mean value of a Poisson distribution. Therefore, one must generate a sequence of 100 Poisson numbers with mean 20 , for example $\{17,15,20,30,16,15,17,23,19,18$, $17,20,16,19,19,17,29 \ldots\}$. Each repetition uses a Poisson number of planes, say, first uses 17 , second 15 , third 20 , fourth 30, and so on. Once the number of planes is chosen, say 17 planes, these parallel planes are randomly located along the $x_{3}$ axes. After that, one must select the number of nuclei per plane with a mean number of nuclei equal to 10 . Again, one generates a sequence of Poisson numbers with mean equal to 10 , for example, $\{10,13,7,6,9,6,8, \ldots\}$. Now, for each plane, one takes the number of nuclei according to the sequence of Poisson numbers. The first plane 10 nuclei, the second plane 13 nuclei, and so on. If one does not do this, the simulation may not coincide with the analytical solution because the analytical solution was derived for both the number of planes and the number of nuclei per plane being Poisson point processes. Notice that the original Cahn's derivation does not mention any of those considerations. In more straightforward cases, for example, when one only has spheres nucleation on uniform randomly located in space, it is possible to obtain good results with a small number of repetitions. Even only one simulation in which the number of nuclei is equal to the mean value of the Poisson distribution may give an acceptable result. But whenever one simulates more complex cases in which more than one Poisson process is present, as the present simulation, it is essential to use the methodology described above.

Another technical point that is useful to comment is the matrix size and processing time. In most of the simulations done by our research group, we found that a matrix $300 \times 300 \times 300$ was enough. Nonetheless, for ellipsoids, the result is not so good because of the large interface area of the ellipsoids. To describe this area accurately, one needs a more refined mesh, thus the necessity to use $500 \times 500 \times 500$ or even $1000 \times 1000 \times 1000$, which requires computational power.

Here, one obtained an exact mathematical solution for transformations in which ellipsoids nucleate on random parallel planes. A heavily deformed rolled polycrystal is going to have most of its interfacial area parallel to the rolling plane. Thus, to a first approximation, one may approximate the nucleation on the interfaces of this rolled polycrystal by nucleation on random parallel planes. This is one interesting application of nucleation of random parallel planes.

At first sight, our main result appears to be overly restrictive. Of more interest is the nucleation on random planes, as initially thought by Cahn. As mentioned above, 
Rios and Villa ${ }^{13,14}$ demonstrated that, for spherical growth, the expression derived for nucleation on random parallel planes is the same as the expression for nucleation on random planes. Rios and Villa ${ }^{13}$ confirmed this by computer simulation.

Therefore, it is reasonable to expect that for ellipsoidal growth, the expression for nucleation on random parallel planes is the same for the expression on random planes. Strictly speaking, this is not entirely true. The problem is the same for ellipsoidal growth nucleated on nuclei located in space according to a Poisson point process. It is well-known ${ }^{15-17}$ that when ellipsoids are aligned, that is, their equivalent axes are parallel; there is an exact expression, see, for example, refs. ${ }^{17,21}$. By contrast, when the orientation of the ellipsoids is random, the exact expression is not valid anymore. This is so because of the issue of "blocking" ${ }^{16,32}$. Godiksen et al. ${ }^{16}$ conducted a computer simulation of randomly oriented ellipsoids. They determined that the exact expression was only valid if the ratio of the longest axes to the shortest axes of the ellipsoid was equal or smaller than four.

In summary, the main objective of this paper was to simulate transformations nucleated on random parallel planes with ellipsoidal growth. Then, to compare the simulations with the new Cahn's model modified for ellipsoids. Furthermore, based on previous work, we can propose that the expression would be identical to the expression for transformations nucleated on random planes with ellipsoidal growth random planes. Unfortunately, although this is valid for spherical growth, for ellipsoidal growth, one must consider the issue of "blocking." Blocking occurs when ellipsoids are located in space so that their axes are not parallel but randomly oriented in space. Blocking interferes with the independence of the growth of individual regions so that the derived expressions are not valid anymore. Nonetheless, previous computer simulation by Godiksen et al. suggests that our expression, when applied to random planes, is valid at least for ellipsoids that have a ratio of the longest to the shortest axis equal to four ${ }^{16,17}$.

\section{Conclusions}

In early papers, Bradley et al. ${ }^{18-20}$ demonstrated that a grain boundary nucleated ferrite allotriomorph is best described by an oblate ellipsoid. In the present paper, we followed up on this idea. We used computer simulation and an exact analytical solution to model nucleation and growth of ellipsoids on random parallel planes. We can draw the following conclusions:

The analytical expression for transformations in which ellipsoids nucleate on random parallel planes agrees very well with computer simulation.

- Computer simulation goes beyond the analytical result. Computer simulation generates the microstructures resulting from nucleation and growth of ellipsoids on random parallel planes.

- Computer simulation transformation kinetics show excellent agreement with exact analytical expressions as one might expect. Nonetheless, this good agreement can only be obtained if the number of nuclei and the number of nuclei per plane are chosen according to a Poisson process. Moreover, each result displayed in the figures is a mean of one hundred simulations. For details, see the discussion section.

- The contiguity showed to be very useful to understand the effect of ellipsoid shape and the number of nuclei per plane on the microstructure. The contiguity revealed a tendency towards "clustering" typical of nucleation on grain boundaries.

- As discussed in detail, the expression derived for random parallel planes is also valid for nucleation on random planes. However, for ellipsoids, owing to the issue of "blocking," it is reasonable to expect that the result will be valid for ellipsoids in which the ratio of the longest to the shortest axis is equal or less than four ${ }^{16}$. For ellipsoids in which the ratio of the longest to the shortest axis is larger than four, the analytical expression can still be applied. However, the analytical expression is going to be progressively less accurate as the ratio of the longest to the shortest axis becomes larger.

\section{Acknowledgments}

This study was financed in part by the Coordenação de Aperfeiçoamento de Pessoal de Nível Superior - Brasil (CAPES) - Finance Code 001. The authors are grateful to Conselho Nacional de Desenvolvimento Científico e Tecnológico, CNPq and Fundação de Amparo à Pesquisa do Estado do Rio de Janeiro, FAPERJ, for the financial support. The authors gratefully acknowledge the Center for Computational Materials Science, Institute for Materials Research, Tohoku University for the use of MASAMUNE-IMR (Materials science Supercomputing system for Advanced Multi-scale simulations towards Next-generation -Institute for Materials Research) supercomputer. In particular, the authors are grateful to Professors Yayoi Terada and Tetsuo Mohri for their collaboration through the Global Institute for Materials Research Tohoku (GIMRT) program.

\section{References}

1. Kolmogorov N. On the statistical theory of metal crystallization. Izv. Akad. Nauk SSSR. Ser. Math. 1937;1(3):355-9.

2. Johnson WA, Mehl RF. Reaction kinetics in processes of nucleation and growth. Trans Metall Soc AIME. 1939;135:416-41.

3. Avrami M. Kinetics of phase change. I: general theory. J Chem Phys. 1939;7:1103-12.

4. Vandermeer RA, Rath BB. Modeling recystallization kinetics in a deformed iron single crystal. Metall Trans, A, Phys Metall Mater Sci. 1989;20:391-401.

5. Rios PR, Villa E. Transformation kinetics for inhomogeneous nucleation. Acta Mater. 2009;57:1199-208.

6. Chiu SN, Stoyan D, Kendall WS, Mecke J. Stochastic geometry and its applications. 3rd ed. Chichester, UK: Wiley; 2013.

7. Rios PR, Villa E. Transformation kinetics for nucleus clusters. Acta Mater. 2009;57:3714-24.

8. Rios PR, Villa E. Transformation kinetics for surface and bulk nucleation. Acta Mater. 2010;58:2752-68.

9. Cahn JW. The kinetics of grain boundary nucleated reactions. Acta Metall. 1956;4:449-59.

10. Fonseca GD, Siqueira FS, Alves ALM, Assis WLS, Rios PR. Computer simulation of site saturation and constant nucleation rate transformations on a network of Kelvin polyhedra. J. Mater. Res. Technol. 2019;8:4534-9. 
11. Barmak KA. A Commentary on: "Reaction kinetics in processes of nucleation and growth. Metall Mater Trans, A Phys Metall Mater Sci. 2010;41A:2711-2.

12. Jägle EA, Mittemeijer EJ. The kinetics of grain-boundary nucleated phase transformations: simulations and modelling. Acta Mater. 2011;59:5775-86.

13. Rios PR, Villa E, Assis WLS, Ribeiro TCS. Kinetics of transformations nucleated on random parallel planes: Analytical modelling and computer simulation. Model. Simul. Mater. Sci. Eng. 2012;20:1-12.

14. Rios PR, Villa E. Transformation kinetics for nucleation on random planes and lines. Image Anal Stereol. 2011;30:153-65.

15. Vandermeer RA, Masumura RA, Rath BB. Microstructural paths of shape-preserved nucleation and growth transformations. Acta Metall Mater. 1991;39:383-9.

16. Godiksen RB, Rios PR, Vandermeer RA, Schmidt S, Juul Jensen D. Three-dimensional geometric simulations of random anisotropic growth during transformation phenomena. Scr Mater. 2008;58:279-82.

17. Sá GMS, Lyrio MS, Alves ALM, Ventura HS, Assis WLS, Villa E, et al. Generalizing Ellipsoidal Growth. Materials Research. 2019;22(3):1-8.

18. Bradley JR, Aaronson HI. The stereology of grain boundary allotriomorphs. Metall Trans, A, Phys Metall Mater Sci. 1977;8A:317-22.

19. Bradley JR, Rigsbee JM, Aaronson HI. Growth kinetics of grain boundary ferrite allotriomorphs in Fe-C alloys. Metall Trans, A, Phys Metall Mater Sci. 1977;8A:323-33.

20. Bradley JR, Aaronson HI. Growth kinetics of grain boundary ferrite allotriomorphs in Fe-C-X alloys. Metall Trans, A, Phys Metall Mater Sci. 1981;12:1729-41.

21. Villa E, Rios PR. On volume and surface densities of dynamical germ-grain models with ellipsoidal growth : a rigorous approach with applications to Materials Science models with ellipsoidal growth: A rigorous approach with. Stoch. Anal. Appl. 2020:122. http://dx.doi.org/10.1080/07362994.2020.1773276.
22. Enomoto M, Aaronson HI. Nucleation kinetics of proeutectoid ferrite at austenite grain boundaries in Fe-C-X alloys. Metall Trans, A, Phys Metall Mater Sci. 1986;17A:1385-97.

23. Tanaka T, Aaronson HI, Enomoto M. Nucleation kinetics of proeutectoid ferrite at austenite grain boundaries in Fe-C-Mn- $\mathrm{X}_{2}$ Alloys. Metall. Trans. A. 1995;26:561-80.

24. Obara T, Lange WF, Aaronson HI, Dom B. Proceedings of an international conference on solid-solid phase transformations. Warrendale, PA: Metallurgical Society of AIME; 1982. p. 1105-09.

25. Cahn JW. The time cone method for nucleation and growth kinetics on a finite domain. Proc MRS. 1996;398:425-37.

26. Ventura HS, Alves ALM, Assis WLS, Rios PR. influence of an exclusion radius around each nucleus on the microstructure and transformation kinetics. Materialia. 2018;2:167-75.

27. Ventura HS, Sá GMS, Duarte ACL, Assis WLS, Rios PR. Computer simulation in 3D of a phase transformation nucleated by simple sequential inhibition process. J. Mater. Res. Technol. 2020;9:152-61.

28. Rios PR, Godiksen RB, Schmidt S, Juul Jensen D. Analytical expression for the evolution of interfacial area density between transformed grains during nucleation and growth transformations. Scr Mater. 2006;54:1509-13.

29. Rios PR, Pereira LO, Oliveira FF, Assis WLS, Castro JA. Impingement function for nucleation on non-random sites. Acta Mater. 2007;55:4339-48.

30. Salazar TC, Assis WLS, Rios PR. Simulation of recrystallization in iron single crystals. Mater Res. 2008;11:109-15.

31. Fonseca GD, Siqueira FS, Alves ALM, Assis WLS, Rios PR. Microstructural descriptors to characterize computer simulated microstructures generated by nucleation on a Kelvin polyhedra network. J. Mater. Res. Technol. 2018;7:337-41.

32. Vandermeer RA. Microstructural descriptors and the effects of nuclei clustering on recrystallization path kinetics. Acta Mater. 2005;53:1449. 